Artigo

\title{
Evaluación de la Estabilidad Atmosférica Bajo Condiciones Físicas y Meteorólogicas del Altiplano Ecuatoriano
}

\author{
Arquímides Xavier Haro Velasteguî ${ }^{1,2}$ (iD, Cecilia Teresa Limáico Nieto², \\ Nelly Patricia Perugachi Cahueñas ${ }^{1}$, María Isabel Fernández Parra ${ }^{3}$ \\ ${ }^{1}$ Carrera de Física, Ingeniería Industrial, \\ Escuela Superior Politécnica de Chimborazo, Riobamba, Ecuador. \\ ${ }^{2}$ Facultad de Ingeniería, Universidad nacional de Chimborazo, Riobamba, Ecuador. \\ ${ }^{3}$ Departamento de Energía y Refrigeración, Escuela de Ingeniería Mecánica, \\ Universidad de Oriente, Santiago de Cuba, Cuba.
}

Received: September 22, 2016 - Accepted: March 24, 2018

\begin{abstract}
Resumen
La estabilidad atmosférica en la capa superficial, influye en muchos de los procesos dinámicos que se dan en la atmósfera, afectan los fenómenos de difusión y transporte de energía y materia, razón por lo que se ha estudiado ampliamente en latitudes medias; sin embargo, existen muy pocos trabajos en condiciones típicas del altiplano ecuatoriano (latitud cero y altura media respecto al nivel del mar de alrededor de $2500 \mathrm{~m}$ ). En el presente trabajo se desarrolla un estudio de la variación de la estabilidad atmosférica en dichas condiciones, usado datos de la estación meteorológica de la Universidad Nacional de Chimborazo del año 2015. Los resultados obtenidos en este estudio muestran un predominio del estado muy estable durante la noche y entre muy inestable e inestable durante el día según la clasificación de Pasquill, con un comportamiento medio mensual regular a lo largo del año.

Palabras clave: estabilidad atmosférica, longitud de Obukhov, dinámica atmosférica, micrometeorología, altiplano, zona ecuatorial.
\end{abstract}

\section{Evaluation of Atmospheric Stability in Physical and Weather Conditions the Highlands Ecuatoriano}

\begin{abstract}
The atmospheric stability in the surface layer, influences many of the dynamic processes that occur in the atmosphere, affect the phenomena of diffusion and transport of energy and matter, reason why it has been studied extensively in middle latitudes, however, There are very few jobs in conditions typical of the Ecuadorian highlands (zero latitude and average height with respect to sea level of around $2500 \mathrm{~m}$ ). In the present work a study of the variation of the atmospheric stability in those conditions is carried out, used data of the meteorological station of the National University of Chimborazo, taken by hours during the year 2015. The results obtained in this study show a predominance of the Very stable during the night and between very unstable and unstable during the day according to the classification of Pasquill, with a regular monthly average behavior throughout the year.
\end{abstract}

Keywords: atmospheric stability, Obukhov length, atmospheric dynamics, micrometeorology, equatorial zone.

\section{Introducción}

La capa límite atmosférica (CLA) representa una pequeña fracción de la atmósfera, comprende aproximadamente el primer kilómetro de la atmósfera. Los procesos de pequeña escala que en ella ocurren son provechosos para muchas de las actividades humanas y, además, son importantes para la existencia y supervivencia de la vida en la tierra.

El aire en la CLA está en continuo movimiento turbulento causando un eficiente intercambio de calor sensible y de calor latente, de cantidad de movimiento y de masa entre 
la superficie terrestre y la atmósfera, y, por tanto, moderando el microclima próximo al suelo.

En la CLA se producen procesos dinámicos importantes debido a la interacción aire-suelo o aire-mar. Podría así decirse que se forma como consecuencia de las interacciones entre la atmósfera y el suelo, produce los flujos de energía en sus diferentes formas y que son fundamentales en los procesos dinámicos y afectan todas las actividades que en dicho lugar se producen (Cian y Waltson, 2014).

En general se considera que el flujo en este nivel es completamente turbulento, de manera que en cualquier punto de ella se observan típicamente variaciones bruscas en el valor de la velocidad, la presión, la temperatura o la humedad del aire. El efecto de la fricción superficial, el calentamiento del suelo y la evaporación se transmite a toda la capa límite atmosférica de forma rápida y eficiente como consecuencia del mecanismo de mezcla turbulenta (Garratt, 1994).

La CLA abarca sólo una pequeña fracción de la atmósfera, no obstante, los procesos a pequeña escala que tienen lugar dentro de esta capa son muy importantes. Así, por ejemplo, cabe señalar que casi toda la energía involucrada en los fenómenos meteorológicos a gran escala y en la circulación general de la atmósfera surge o se transmite a través de la CLA. La atmósfera recibe del suelo la mayor parte de calor (Teixeira, 2008).

La estabilidad atmosférica es una condición de equilibrio. De forma que, en una atmosfera estable se produce una perturbación en el flujo. Si tras la perturbación vuelve al estado original el sistema es estable. Pero si la perturbación evoluciona y cambia el estado del sistema entonces se dice que es inestable, existen varios métodos para establecer el grado de estabilidad atmosférica, siendo el más usados aquel que se basa en las propuestas hechas por Pasquill, Obukhov y Van Ulden - Hostlang, los cuales para este trabajo se han adaptado a las condiciones de la zona de estudio (entre $5^{\circ}$ latitud sur y $1^{\circ} 21^{\prime}$ latitud norte y alrededor de, $78^{\circ}$ longitud oeste) (Van Ulden, 1985), con la finalidade de estudiar su comportamento durante el día y los diferentes meses del año.

Para realizar el trabajo se han considerado las condiciones típicas del altiplano ecuatoriano, éste está formado, por un conjunto de mesetas que forma la cordillera de Los Andes. Se encuentra ubicada entre el Nudo de los Pastos al norte hasta el de Loja al sur, ocupando una franja de $800 \mathrm{~km}$ de largo y alrededor de $50 \mathrm{~km}$ de ancho, la altura fluctúa entre 1000 m.s.n.m. y 6310 m.s.n.m., con un promedio de alrededor de 2500 m.s.n.m., una temperatura promedio de $14{ }^{\circ} \mathrm{C}$

La ciudad de Riobamba, ubicada a $1^{\circ} 38^{\prime} 3^{\prime \prime}$ y $1^{\circ} 4^{\prime}$ de latitud sur, $78^{\circ} 39^{\prime}$ y $78^{\circ} 40^{\prime} 36^{\prime \prime}$ de longitud oeste, meseta a $2750 \mathrm{~m}$ de altura sobre el nivel del mar, rodeada de altas montañas a $188 \mathrm{~km}$ al sur de la ciudad de Quito, para el desarrollo del trabajo se usan datos de la estación meteorológica de la Universidad Nacional de Chimborazo, que pertenece al proyecto "Programa integrado de control de monitoreo de la calidad del aire en la ciudad de Riobamba usando modelos matemáticos", de tipo Vaisala estandarizada a las normas internacionales, automática, adquirida e instalada en abril del 2014, con monitoreos de $1 \mathrm{~h}$ a lo largo del año 2015 (Haro et al., 2014; Limaico, 2004).

\section{Materiales y Métodos}

\subsection{Dinámica atmosférica}

La mayor parte de los fenómenos de la atmósfera se desenvuelven en la troposfera y particularmente en la CLA, estos procesos se deben al desarrollo de la turbulencia mecánica asociada con el rozamiento del viento con la superficie sólida y la turbulencia convectiva, producida por el flujo de calor entre el suelo y el aire, que se produce por el calentamiento del suelo en las horas del día (Rodriguez y Portela, 2004).

La estabilidad atmosférica se relaciona con la dinámica del sistema atmósfera-suelo, y puede analizarse a través de ciertos parámetros, como la turbulencia convectiva producida por los flujos térmicos influenciados por el grado de nubosidad, balance de radiación, gradiente de temperatura, principalmente y la turbulencia mecánica debida a la rugosidad superficial y viento transversal (Reyes, 2001).

La mayor parte de procesos que ocurren en la atmosfera son de carácter turbulento, es decir un sistema no lineal, que no responde a las ecuaciones dinámicas planteadas en la física clásica, las cuales describen esencialmente sistemas lineales. Por esta razón, se han realizado una serie de aproximaciones (modelos) que permiten describir con cierta aproximación la dinámica de la atmosfera, los cuales se han desarrollado básicamente a latitudes medias, siendo entre los más conocidos el de Van Ulden and Hostlag (Van Ulden, 1985) y Obukhov (Mcnaughton, 2009), trabajos que se toman de base para este estudio (Haro et al., 2014).

\subsection{Flujos superficiales en la capa límite atmosférica}

Usando métodos propuestos básicamente por Van Ulden y Hostlag se ha desarrollado un estudio de flujos superficiales en la capa límite atmosférica de la ciudad de Riobamba. Para lo cual se considera que cuando una masa de aire circula sobre una superficie y pasa a otra con características diferentes, sus propiedades físicas cambian. Los parámetros básicos que describen las condiciones del aire, tales como la temperatura y la humedad, comienzan su transformación en la superficie de contacto, mediante una serie de procesos se propagan a capas de mayor altura; entonces, a esa porción de la atmósfera donde se observa el efecto directo de la superficie terrestre se la define como capa límite. En ella se transfiere energía desde la superficie a la atmósfera y viceversa, mediante flujos turbulentos de calor sensible $\left(Q_{H}\right)$, latente $\left(Q_{E}\right)$ y de cantidad de movimiento. Para entender estos procesos en mayor detalle se 
describe algunos de los aspectos que influyen en este proceso (Van Ulden y Holtslag,1985).

\subsection{Balance de energía superficial}

En este método se considera el balance total de energía correspondiente a un elemento de volumen con base a la superficie, que contiene cobertura vegetal y la atmósfera circundante. Una parte de la energía que recibe se empleará en producir evapotranspiración y es la que interesa evaluar, traduciendo el resultado a unidades de agua evaporada.

La ecuación del balance de energía, de forma simplificada sería, la dada en la Ec. (1):

$$
R_{N}=Q_{H}+Q_{E}+Q_{G}
$$

donde $R_{N}$ flujo de radiación neta, $Q_{G}$ flujo de calor almacenado en el suelo, $Q_{H}$ flujo de calor sensible y $Q_{E}$ flujo de calor latente

En la fórmula no se tiene en cuenta la energía invertida en la fotosíntesis, ya que es despreciable frente a otros flujos energéticos. Asimismo, se desprecian la divergencia horizontal de calor latente y de calor sensible en dicho volumen. También se supone nulo, el flujo de calor advecticio procedente de las zonas circundantes, para cuya hipótesis es preciso crear una zona de amortiguamiento de este efecto (conocido como efecto "oasis") alrededor de la parcela experimental, especialmente si se trabaja en zonas áridas y la parcela se mantiene húmeda artificialmente (por irrigación).

Los términos $R_{N}$ y $Q_{G}$ se pueden medir con bastante aproximación directamente con el pirómetro usando la relación de Kasten y Czeplak (1980), para determinar la nubosidad, con $Q_{G}$ en función de $R_{N}$ De Bruin and Holtslag (1982), con pequeños termopares enterrados en el suelo. En ocasiones también se desprecia el flujo de calor almacenado en el suelo $\left(C_{a}\right)$ lo que puede dar lugar a que se incurran en errores importantes. Por ejemplo, a primeras horas de la mañana $Q_{G}$ puede ser negativo y del orden del $25 \%$ de $R_{N}$ por lo que despreciar este término producirá tanto más error, cuanto más corto sea el intervalo de medida, y más próximo a las horas matinales. Otras veces $C_{a}$ se estima empíricamente como función fija de $R_{N}$ (Aeromod, 2004).

El flujo de calor sensible $\left(Q_{H}\right)$ Ec. (3), y el flujo de calor latente $\left(Q_{L}\right)$ no pueden medirse por separado. El flujo de calor sensible es el calor que calienta el aire en el volumen elemental considerado y el flujo de calor latente, es la energía que se emplea exclusivamente para producir evaporación. El cociente entre ambos flujos $\left(Q_{H} / Q_{L}\right)$ se conoce con el nombre de relación de Bowen (Cain, 2004).

Partiendo de un balance de energía superficial (Van Ulden and Holtslag, 1985):

Con

$$
Q_{G}=a R_{N}
$$

$$
Q_{H}=\left[\frac{(1-\alpha)+S}{1+S}\right] R_{N}(1-a)-\beta
$$

donde $a=0.1$ para áreas rurales y $a=0.3$ para áreas urbanas que el que se tomo en este estudio (Doll et al., 1985). $\beta$ es una constante igual a $20 \mathrm{~W} \mathrm{~m}^{-2}$ y $\alpha$ como 0.75 para ambientes urbanos, el parâmetro $S$ es definido por:

$$
S=e^{0.055(T-279)}
$$

La radiación neta es observada, también puede ser parametrizada, según la Ec. (5), basada en el albedo $A$, ángulo de elevación solar $\theta$, fracción de nubosidad $N$, flujo de radiación incidente $Q$, y la temperatura del aire $T$.

$$
R_{N}=\frac{\left((1-A) Q+c_{1} T^{6}-\sigma T^{4}+c_{2} N\right)}{1+c_{3}}
$$

donde: $\sigma=5.67 \times 10^{-8} \mathrm{~W} \mathrm{~m}^{-2} \mathrm{~K}^{-4} \equiv$ es la constante de Stefan-Boltzmann and $c_{1}=5.31 \times 10^{-13} \mathrm{~W} \mathrm{~m}^{-2} \mathrm{~K}^{-6} \mathrm{y}$ $c_{2}=60 \mathrm{~W} \mathrm{~m}^{-2}$ y $A=0.185\left(1-e^{-\frac{h}{100}}\right)$.

Con $h$ humedad, el parámetro $c_{3}$ es dado por la fórmula:

$$
\begin{gathered}
c_{3}=\frac{0.38((1-\alpha) S+1)}{S+1} \\
Q=\left(a_{1} \sin \phi+a_{2}\right)\left(1-b_{1} N^{b_{2}}\right) \\
a_{1}=990 \mathrm{~W} \mathrm{~m}^{-2}, a_{2}=-30 \mathrm{~W} \mathrm{~m}^{-2}, b_{1}=0.75, b_{2}=3.4 .
\end{gathered}
$$

Con $\phi$ elevación solar, que se puede calcular en función de la latitud, longitud de la zona de estudio y el día juliano (Shellers, 1965).

De cuya relación se determina la nubosidad aproximada (Aeromod, 2004).

$$
N=\left(1-\frac{b_{1} Q}{a_{1} \sin \phi+a_{2}}\right)
$$

\subsection{Método De Monin-Obukhov}

Aplicando la teoría de similaridad de Monin-Obukhov se puede determinar la velocidad de fricción $u_{*}$ en función de la velocidad del viento $U_{z}$ y la atura $z$.

$$
u_{*}=k U_{z}\left[\ln \left(\frac{z}{z_{0}}\right)-\psi_{M}\left(\frac{z}{L}\right)+\psi_{M}\left(\frac{z_{0}}{L}\right)\right]^{-1}
$$

Con $k$ constante de Von Karman $(k=0.4), z_{0}$ largo de rugosidad superficial del lugar $\left(z_{0}=0.5 \mathrm{~m}\right)$ que se determina mediante tablas (Marrero, 2011).

$$
L=-\frac{\rho C_{p} T u_{*}^{3}}{k g Q_{H}}
$$

$L$ longitud de Obukhov, con $T$ temperatura del aire, g constante de aceleración de gravedad, $\rho$ densidad del aire, 
$C_{p}$ calor específico del aire a presión constante y $Q_{H}$ flujo de calor sensible.

La función de estabilidad $\psi_{M}$ se determina por $L<0$ (Inestable), como:

$$
\psi_{M}=2 \ln \left(\frac{1+x}{2}\right)+\ln \left(\frac{1+x^{2}}{2}\right)-2 \tan ^{-1}(x)+\frac{\pi}{2}
$$

De las Ecs. (9) y (10) se puede hallar la velocidad de fricción y la longitud de Obukhov por interacción tomándose inicialmente $\psi_{M}=0$ y $L=\infty$ (Wyngaard, 1998) hasta alcanzar una variación aproximada del $1 \%$ de dos valores sucesivos de $L$ para valore mayores de cero y menores de cero y con el mismo criterio, para el caso estable, $L>0$ se aplica la relación (Panofsky and Dutton, 1984).

$$
\psi_{M}=-5\left(\frac{z}{L}\right)
$$

donde

$$
x=\left(1-16 \frac{z}{L}\right)^{\frac{1}{4}}
$$

\subsection{Estabilidad atmosférica}

La estabilidad atmosférica se puede asociar al gradiente de temperatura, comparando con el gradiente de temperatura ambiental es el gradiente de temperatura seca adiabática. Bajo condiciones adiabáticas, un volumen templado de aire ascendente se comporta como un globo. Se expandirá hasta que su densidad se iguale con la del aire circundante (Sang et al., 2009).

El aire seco se expande a una velocidad de 9.8 Grados Centígrados por cada 100 metros de altura. Una atmósfera Neutralmente Estable tiene lugar cuando el gradiente de temperatura ambiental (GTA), es igual al gradiente de temperatura seca Adiabática (GTSA) o lo que es lo mismo la velocidad de enfriamiento es igual aproximadamente 1 grado centígrado cada $100 \mathrm{~m}$.

Una Atmósfera Inestable se da cuando el gradiente de temperatura ambiental excede del gradiente de temperatura seca adiabática, o lo que es lo mismo la velocidad de enfriamiento en la ascensión es mayor a 1 grado centígrado por cada $100 \mathrm{~m}$.

Una Atmósfera Estable tiene lugar cuando el gradiente de temperatura ambiente es menor que el gradiente de temperatura seca adiabática, o lo que es lo mismo la velocidad de enfriamiento, es menor a 1 grado centígrado $/ 100 \mathrm{~m}$.

En la Tabla 1 se presenta los limites asociados a las clases de estabilidad de Pasquill tomando como referencia el largo de Monin - Obukhov $(L)$ calculado para $z_{0}=0.5 \mathrm{~m}$ y $z=10 \mathrm{~m}$ (Floors, 2011).

\subsection{Corrección de parámetros físicos}

El comportamiento del aire no es constante con la temperatura, presión o humedad, para el caso del altiplano
Tabela 1 - Correspondecia entre la longitud de Monin - Obukhov y las clases de estabilidad de Pasquill.

\begin{tabular}{lc}
\hline Clases de Pasquill & $1 / L$ \\
\hline A Muy inestable & $\frac{1}{L}<-0.056$ \\
B Inestable & $-0.056 \leq \frac{1}{L}<-0.016$ \\
C Moderadamente inestable & $-0.016 \leq \frac{1}{L}<-0.004$ \\
D Neutra & $-0.004 \leq \frac{1}{L}<0.002$ \\
E Moderadamente estable & $0.002 \leq \frac{1}{L}<0.006$ \\
F Estable & $0.006 \leq \frac{1}{L}<0.022$ \\
G Extremadamente estable & $\frac{1}{L} \geq 0.022$ \\
\hline
\end{tabular}

ecuatoriano, estas variaciones pueden ser representativas, por lo que se procede a realizar una corrección de los mismos basado en la teoría termodinâmica (Haro, 2004).

Calor específico del aire $C_{e}(\mathrm{~J} / \mathrm{kg} . \mathrm{K})$

$$
C_{e}=999.2+0.1434 T+1.101 \times 10^{-4} T^{2}
$$$$
-6.7581 \times 10^{-8} T^{3}
$$

$T$ temperatura ambiente.

$$
\begin{aligned}
& \text { Densidad } \rho\left(\mathrm{kg} / \mathrm{m}^{3}\right) \\
& \rho=\frac{0.34848 p-0.009 h_{r} e^{0.061 T}}{273.15+T}
\end{aligned}
$$

$h_{r}=$ humedad relativa en el aire en $\%, p=$ presión atmosférica local (mbar).

Que en condiciones normales se puede definir $\rho=\frac{353.44}{T+273.5}$.

\section{Resultados y Discusión}

En la Tabla 2 se observan los parámetros promedios, mínimos y máximos de los parâmetros meteorológicos que se usaron para el estúdio de la variabilidad de la estabilidad atmosférica, en la cual se puede observar, que los valores de temperatura y presión varían significativamente respecto a condiciones normales de la zona (Ecuatorial), debido a la altura respecto al nivel del mar, donde la presión no tiene una fluctuación significativa respecto a su media, pero si lo tiene respecto al nivel del mar (1013.21 mbar).

Otro de los parámetros que se deben observar es la temperatura (Tabla 2), que fluctúa entre los $24.87{ }^{\circ} \mathrm{C}$ a $3.86{ }^{\circ} \mathrm{C}$, naturalmente para la latitud de la zona de estudio

Tabela 2 - Parámetros meteorológicos promedios, mínimos y máximos, calculados en la ciudad de Riobamba.

\begin{tabular}{lccc}
\hline Parámetro & Promedio & Mínimo & Máximo \\
\hline Temperatura $\left({ }^{\circ} \mathrm{C}\right)$ & 13.55 & 3.86 & 24.87 \\
Humedad $(\%)$ & 76.47 & 20.74 & 99.67 \\
Presión $(\mathrm{mbar})$ & 731.54 & 726.39 & 735.91 \\
Radiación incidente $\left(\mathrm{W} / \mathrm{m}^{2}\right)$ & 224.39 & 0.00 & 1276.87 \\
Velocidad del viento $(\mathrm{m} / \mathrm{s})$ & 1.39 & 0.00 & 7.95 \\
\hline
\end{tabular}


es demasiado baja, sin embargo se debe recordar que esta sobre los $2700 \mathrm{~m}$ sobre el nivel del mar, que produce un descenso natural de la temperatura con la altura.

La radiación presenta una alta intensidad (Tabla 2) alcanzando los $1276.87 \mathrm{~W} / \mathrm{m}^{2}$, con regularidad a lo largo del año, lo que produce flujos regulares de energía entre el aire y el suelo, dada su posición geográfica, la velocidad del viento y humedad no se observan cambios notorios, lo que permite deducir, que al depender la dinámica atmosférica de estos parâmetros, la misma será afectada.

Con los datos de la estación meteorológica, medidos cada hora durante el año 2015 y aplicando el modelo de Van Ulden Hostlang, a cuál se le hace un ajuste de los parámetros físicos de acuerdo a las condiciones de la zona, Ecs. (14) y (15), se calculan los parámetros micrometeorológicos necesarios para determinar la estabilidad atmosférica (Tabla 3).

En la Tabla 3 se presentan los resultados de los parámetros micrometeorológicos, promedio, máximo y mínimo de las variables de flujo de calor sensible, flujo de calor latente, flujo de calor superficial, velocidad de fricción y longitud de Obukhov.

Los flujos superficiales determinados muestran un comportamiento similar entre ellos (Tabla 3), mientras la radiación neta alcanza valores que llegan a los $842.90 \mathrm{~W} / \mathrm{m}^{2}$, la velocidad de fricción varia hasta $1.10 \mathrm{~m} / \mathrm{s}$ aproximadamente y la longitud de Obukhov presenta varia-

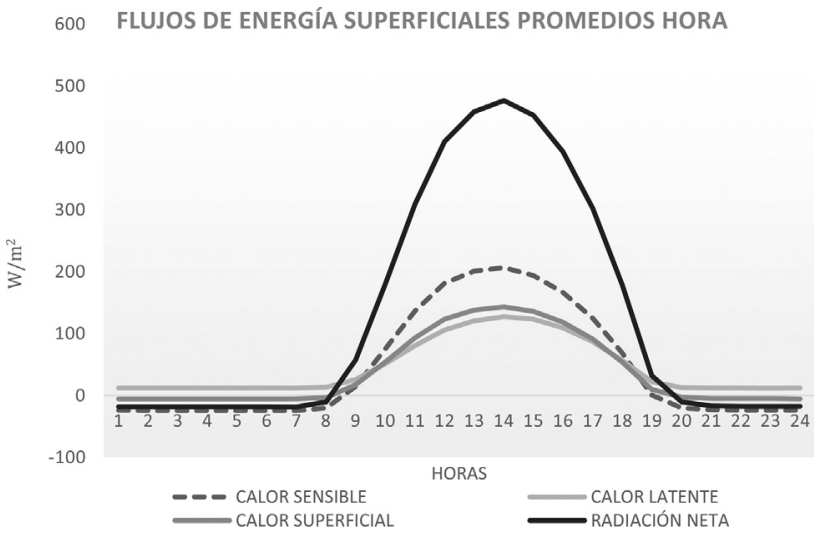

Figura 1 - Flujos de energía superficial, calculado promedio hora en el año 2015 .

ciones significativas alcanzando valores que fluctúan entre -6732.61 y $31020.98 \mathrm{~m}$.

Se debe notar (Tabla 3) que el flujo de calor sensible tiene un superior respecto al calor latente y flujo de calor superficial, entre las $9 \mathrm{~h} 00$ y $18 \mathrm{~h} 00$, debido a la acción de la radiación, la cual claramente mantiene la energía en el ambiente, a pesar que rápidamente disminuye en horas de la noche, Fig. 1.

En la Fig. 2 se presenta los resultados de la velocidad de fricción en promedio por hora para el año 2015, se puede observar un comportamiento regular durante la noche que

\section{VELOCIDAD DE FRICCIÓN PROMEDIO HORAS EN LA CIUDAD DE RIOBAMBA}

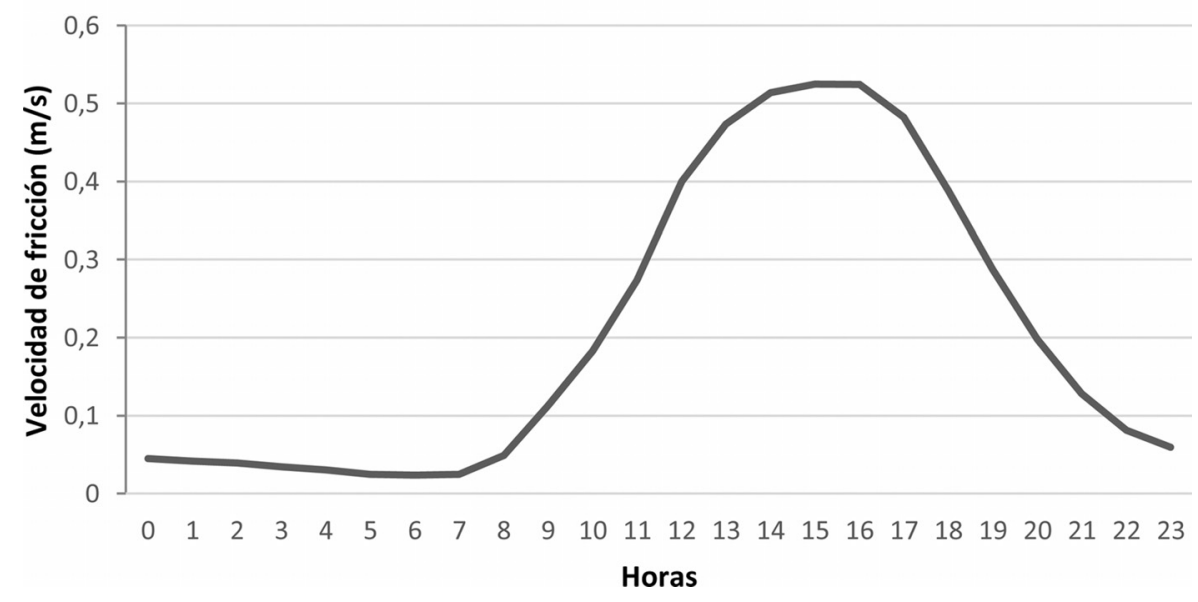

Figura 2 - Velocidad de fricción, calculado promedio hora en el año 2015.

Tabela 3 - Parámetros micrometeorológicos medios calculados bajo condiciones de la zona de estúdio.

\begin{tabular}{lcccccc}
\hline & $\begin{array}{c}\text { Flujo de calor } \\
\text { sensible }\left(\mathrm{W} / \mathrm{m}^{2}\right)\end{array}$ & $\begin{array}{c}\text { Flujo de calor } \\
\text { latente }\left(\mathrm{W} / \mathrm{m}^{2}\right)\end{array}$ & $\begin{array}{c}\text { Flujo de calor } \\
\text { superficial }\left(\mathrm{W} / \mathrm{m}^{2}\right)\end{array}$ & $\begin{array}{c}\text { Radiación neta } \\
\left(\mathrm{W} / \mathrm{m}^{2}\right)\end{array}$ & $\begin{array}{c}\text { Velocidad de } \\
\text { fricción }(\mathrm{m} / \mathrm{s})\end{array}$ & $\begin{array}{c}\text { Longitud de } \\
\text { Obukhov }(\mathrm{m})\end{array}$ \\
\hline Promedio & 44.14 & 44.17 & 37.84 & 126.15 & 0.21 & 4.12 \\
Mínimo & -27.19 & 11.35 & -6.29 & -20.98 & 0.00 & -6732.61 \\
Máximo & 359.76 & 230.27 & 252.87 & 842.90 & 1.10 & 31020.98 \\
\hline
\end{tabular}


se caracteriza por tener velocidades bajas, mientras que en el día esta inicia a crecer a partir de las 6 h00 hasta las 16 h00, se debe recordar que por ser la velocidad que se asocia a la turbulencia mecánica, la misma es muy importante en la estabilidad atmosférica.

La longitud de Obukhov presenta una gran fluctuación entre las horas del día y la noche, Fig. 3, particularmente en la transición de la noche al día se puede observar un pico significativo, lo cual indica la regularidad del comportamiento de la misma a lo largo del año, presentando valores generalmente bajos, que establecen un predo- minio de la estabilidad durante la noche e inestabilidad durante el día, lo cual se verifica en la Fig. 4, en la cual se observa con mayor frecuencia el estado muy estable de la clasificación de Pasquill durante las horas de la noche, y en el día los estados muy inestable, inestable y moderadamente inestable, con un pequeño predominio del muy inestable, mientras que para el estado neutro con menor frecuencia hay una presencia especialmente en horas del día y parte de la noche.

En la Fig. 5 se presenta los resultados de las frecuencias de estabilidad promedio mes, donde se puede observar

LONGITUD DE OBUKOV MEDIA POR HORAS EN LA CIUDAD DE RIOBAMBA

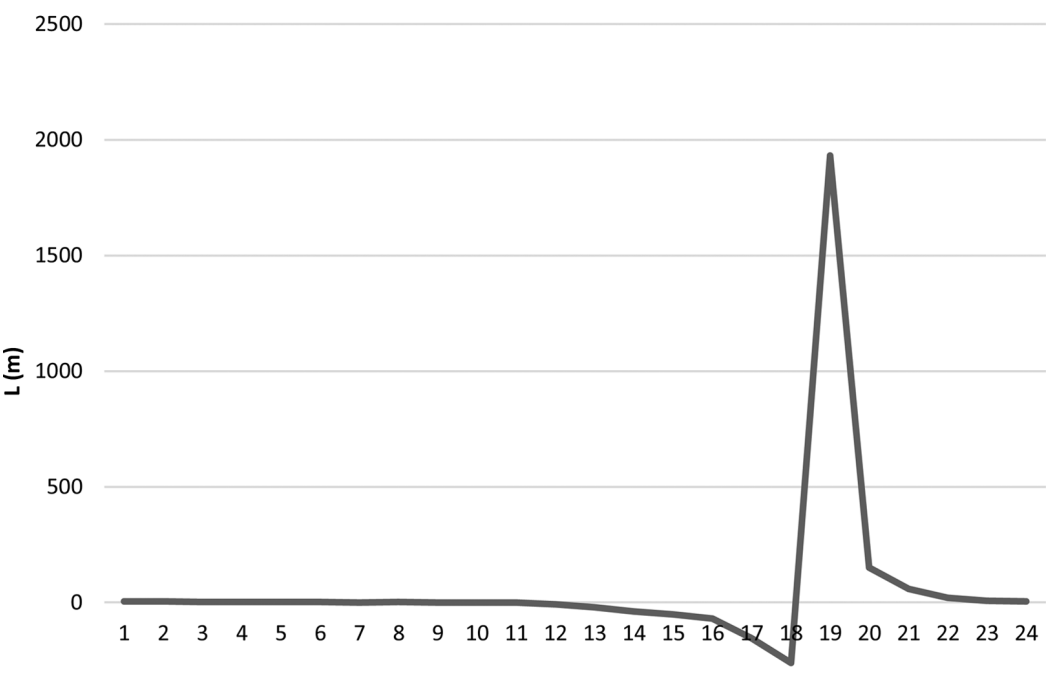

$-500$

Horas

Figura 3 - Longitud de Obukhov, calculado promedio hora en el año 2015.

\section{FRECUENCIA DE ESTABILIDAD POR HORAS ANUAL}

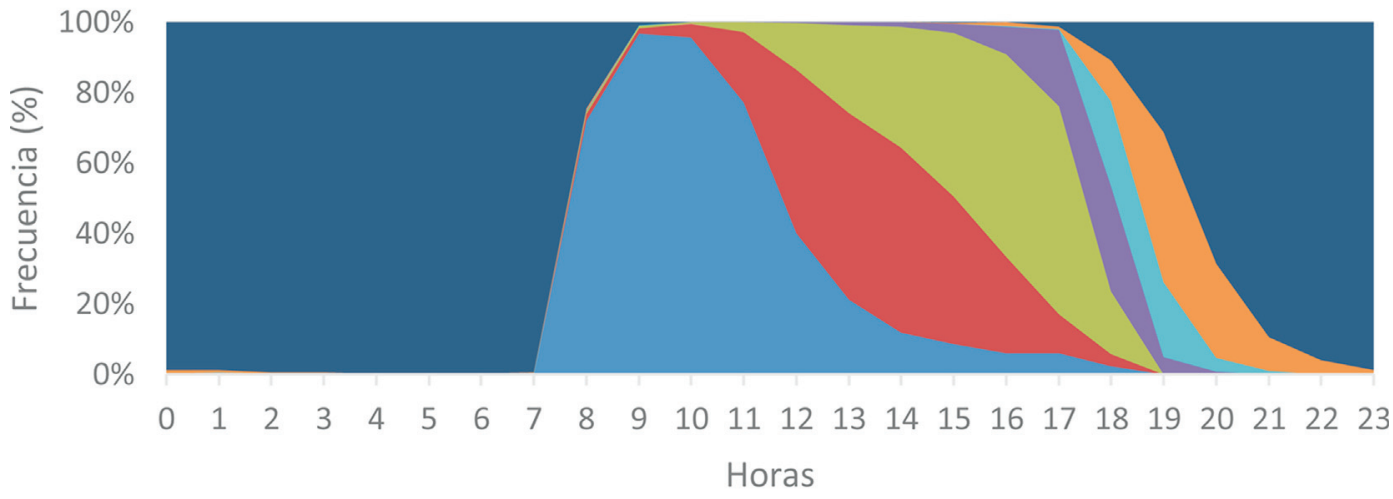

$\begin{array}{lll}\text { Muy Inestable } & \text { Inestable } & \text { Moderadamente Inestable } \\ \text { Neutra } & \text { Ligeramente Estable } & \text { Estable }\end{array}$

Muy Estable

Figura 4 - Gráfico de frecuencias de estabilidad según la clasificación de Pasquill, calculada promedio hora en el año 2015. 


\section{ESTABILIDAD PROMEDIO MENSUAL}

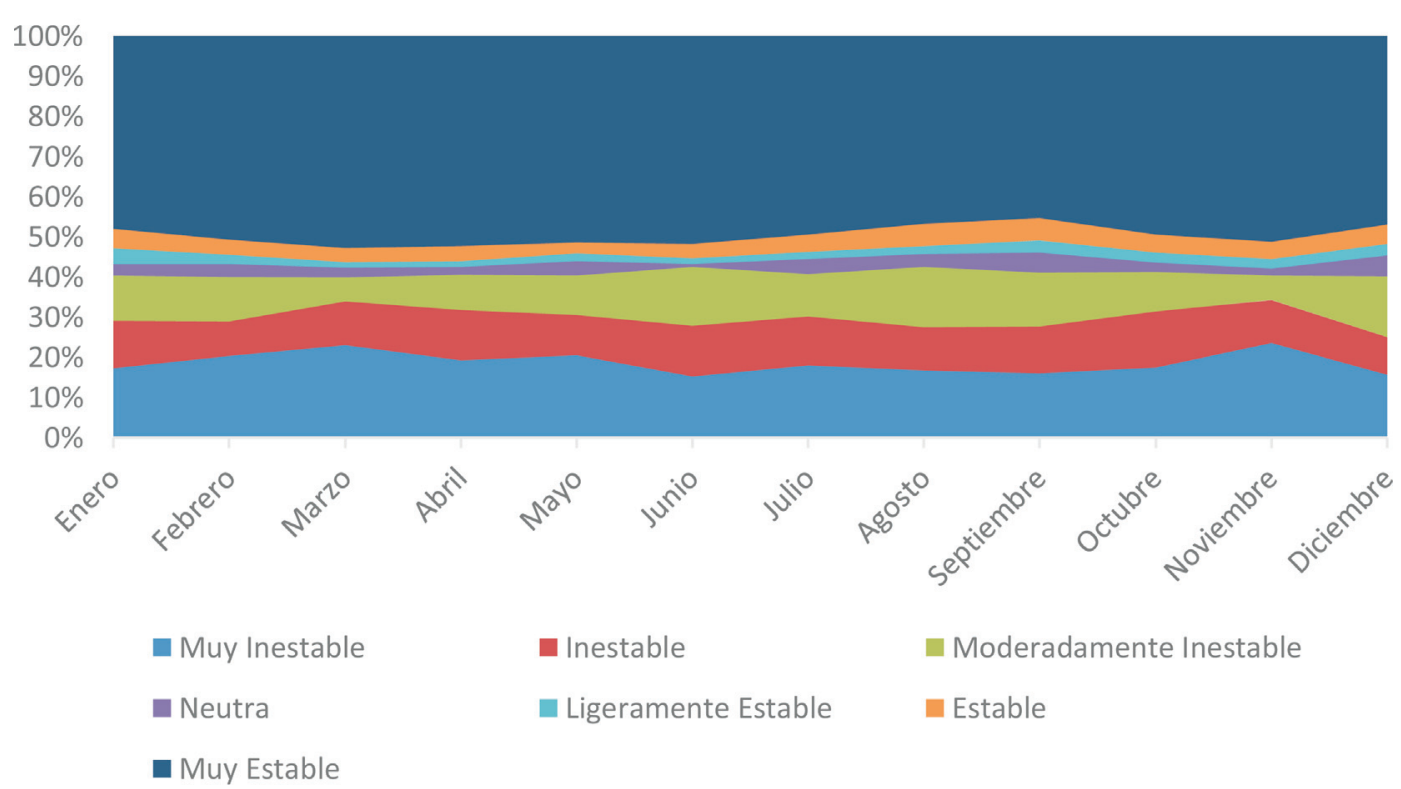

Figura 5 - Gráfico de frecuencias de estabilidad según la clasificación de Pasquill, calculadas promedio mes en el año 2015.

una alta regularidad a lo largo del año del comportamiento de las mismas, dado la posición geográfica de la zona, que permite una regularidad del comportamiento del clima a lo largo del año, con un claro predominio del estado muy estable.

\section{Conclusiones}

Los flujos de calor sensible son predominantes respecto al flujo de calor latente y superficial en horas del día, debido la fuerte incidencia de la radiación solar por la posición geográfica y altura respecto al nivel del mar de la zona, lo cual influye en el comportamiento inestable durante las horas del día.

En las horas de la noche el flujo de calor sensible baja rápidamente, lo que provoca que la atmósfera sufra una inversión en su gradiente de temperatura, manifestándose en el cambio de la estabilidad, con un predominio del estado muy estable según la clasificación de Pasquill.

La frecuencia de estabilidad mensual tienden a mantenerse constante largo del año, dado la posición geográfica de la zona.

\section{Referencias}

AEROMOD, EPA Description of model formulation. EPA454/R-03-004. Office of Air Quality Planning and Standards, U.S.A., 2004

BELJAARS, A.C.M; HOLTSLAG, A.A.M. Flux parametrization overland surface for atmospheric models, Applied Meteorology, v. 30, n. 3, p 327-341, 1991.

BRUN, H.D.R; HOLTSLAG A.A.M. A simple parametrization of de surface fluxes of sensible and latent heat during day time compared whit the Penman Monteinth concept, Applied Meteorology, v.21, p 1610-1621, 1982.

BUSINGER, J.A.; WINGAARD, J.C.; IZUMI Y.; BRADLEY, E.F. Flux-profile relationships in the atmospheric surface layer, Journal of the Atmospheric Sciencies, v. 28, p. 181-189, 1971.

CIAN, J.D.; SIMON W. A study of stability effects in forested terrain. Journal of Physics: Conference Series 555, 2014.

DOLL, D.; CHING, J.K.S.; KANESHIRE, J. Parametrization of surfaces heating for soli and concrete using net radiation data, Boundary Layer Meteorology, v. 32, p. 351-372, 1985.

FINZI, G.; BRUSASCA, G. La qualitá dell'aria; modelli previsionali e gestionali, Masson, Milano, 1991.

GARRATT, J.R. The atmospheric boundary layer, EarthScience Reviews, v. 37, n. 1, p. 89-134, 1994.

HARO, A.X.; LlOSAS, Y.E.; LIMÁICO, C.T. Predicción de datos meteorológicos en cortos intervalos de tiempo en la ciudad de Riobamba usando la teoría del caos, Revista Iberoamericana de Sistemas, Cibernética e Informática, v. 13, n. 1, p. 35-41, 2016.

HARO A.X. Estudio de difusión de contaminantes en el Parque Industrial Riobamba, Informe proyecto, 2001, Riobamba-Ecuador.

KASTEN, F.; CZEPLAK, G. Solar and Terrestrial Radiation dependent on the Amount and Type of Cloud, Solar Energy, v. 24, p. 177-189, 1980.

LIMAICO, C.T. Estudio de las variables micro meteorológicas usando la teoría del caos en la ciudad de Riobamba, Tesis de pregrado, ESPOCH, 2004.

MARRERO, S.M. Parámetros de rugosidad representativos de terrenos naturales, Departamento de Física Aplicada. Universidad de Granada, 2011.

MCNAUGHTON, K. The rise and fall of Monin-Obukhov theory. Asia Flux Newsletter, v. 30, p. 1-4, 2009. 
PANOFSKY, H.A.; DUTTON, J.A. Atmospheric Turbulence Models and Methods for Engineering Applications. New York: Wiley, 1984.

REYES, S. Introducción a la meteorología, Universidad de Baja California, p. 30-50, 2001.

RODRÍGUEZ, R.; CAPA, A.; PORTELA, A. Meteorología y Climatología, FECYT (Fundación Española para la Ciencia y la Tecnología), 2004.

RODRÍGUEZ, V.D.; QUINTERO S.A.; YOSDANY, G.I.; CUESTA S.O.; SÁNCHEZ, D.A. Variación de la estabilidad y altura de la capa de mezcla en la ciudad de Pinar del Río: su relación con condiciones sinópticas, Revista Brasileira de Meteorología, v. 30, n. 1, p. 1-15, 2015.

SANG, J.P.; SOON, U.P.; CHANG, H.H.; MAHRT L. Fluxgradient relationship of water vapor in the surface layer ob- tained from CASES-99 experiment, Journal of Geophysical Researcvh, v. 114, p. 1-12, 2009.

TEIXEIRA, J.; STEVENS, B.; BRETHERTON C.S.; CEDERWALL, R.; DOYLE, J.D.; et al. Parameterization of the Atmospheric Boundary Layer, Bulletin of the American Meteorological Society, v. 89, n. 4, p. 453-458, 2008.

VAN ULDEN, A.P; HOLTSLAG, A,A,M. Estimation of Atmospheric Boundary Layer Parameters for Diffusion Applications, Journal of climate and Applied Metereology, v. 24, n. 11, p. 1196-1207, 1985.

WYNGAARD, J.C. Convection viewed from a turbulence perspective. Buoyant Convection in Geophysicsl Flows, Advanced Science Institute Series, Springer Science \& Business Media, v. 513, p. 23-39, 1998.

This is an Open Access article distributed under the terms of the Creative Commons Attribution Non-Commercial License which permits unrestricted non-commercial use, distribution, and reproduction in any medium provided the original work is properly cited 